\title{
Ipsilateral trans-axillary aortic valve replacement in a patient with ilio-femoral and coronary artery bypass surgery, permanent pacemaker and subclavian stent
}

\author{
Abdullah Aslan ${ }^{1}$, Emrah Uğuz ${ }^{1}$, Esma Arslan ${ }^{1}$, and Tahir Durmaz ${ }^{1}$ \\ ${ }^{1}$ Ministry of Health Ankara City Hospital Cardiovascular Hospital
}

November 5, 2020

\begin{abstract}
Transcatheter aortic valve replacement (TAVR) has become the procedure of choice for patients deemed high-risk or inoperable for surgical aortic valve replacement. For patients with inadequate iliofemoral access, the risk of vascular injury is extremely high and alternative techniques have been utilized and include the transapical, transaortic and transsubclavian or transaxillary $(\mathrm{TAx})$ routes. Alternative approaches often require open surgical exposure under general anesthesia. Herein, we describe a unique TAx TAVR with a balloon-expandable Edwards SAPIEN 3 device in a 77 year-old male patient with the history of coronary artery and ilio-femoral bypass surgery, permanent pacemaker implantation at the left pectoral fossa and left subclavian artery stent.
\end{abstract}

\section{INTRODUCTION}

Transcatheter aortic valve replacement (TAVR) is now established as a viable therapy for the treatment of severe aortic stenosis (AS). Greater than $90 \%$ of TAVR cases in the United States are performed via transfemoral approach at the present time. ${ }^{[1]}$ Despite incrementally smaller TAVR delivery systems, femoral arterial access can be inappropriate due to diminutive vessel caliber, significant calcification and atheroma or severe tortuosity between the arteriotomy site and the native valve. For such patients, an alternative approach to TAVR is required. Until now, multiple approaches have been described, including transapical, transcarotid, transcaval, direct aortic, transsubclavian and transaxillary $(\mathrm{TAx}) \cdot{ }^{[2,3]}$

An axillary artery is less affected by atherosclerotic disease even in the presence of extensive iliofemoral and coronary artery disease. Therefore, TAx route can be preferred for special group of patient. After surgical cutdown of the axillary artery, the sheath and valve can be introduced into the aorta and reach the aortic annulus. This procedure presents some aspects similar to the transfemoral approach with the advantage of avoiding the complication during passage in peripheral arteries, typical of the transfemoral approach, and the invasiveness of the transapical approach, which requires thoracotomy, apical exposure, and passage in the left ventricle. ${ }^{[3,4]}$ Here, we present a 77 year-old male patient with the previously known ilio-femoral and coronary artery bypass surgery, permanent pacemaker implantation at left pectoral fossa and left subclavian artery (SCA) stent who underwent successful TAx TAVR through left axillary artery (LAx) with the balloon-expandable Edwards SAPIEN 3 (S3) valve.

\section{CASE REPORT}

A 77 years-old male admitted to our hospital with the complaint of worsening dyspnea for 2 months (New York Heart Association Class III-IV). His past medical history revealed coronary artery bypass grafting with a left internal mammary artery to the left anterior descending (LIMA-LAD) and a saphenous vein to right coronary artery as well as hypertension, hyperlipidemia, peripheral artery disease with bilateral 
ilio-femoral bypass, left SCA stent (balloon-expandable, non-covered, 8x40-mm Scuba (Invatec S.p.A, Italy) stent) and permanent pacemaker implantation at the left pectoral fossa due to complete heart block. At physical examination, there was a grade $4 / 6$ systolic ejection murmur best heard at aortic focus. Transthoracic echocardiography showed left ventricular systolic dysfunction with an ejection fraction of $45 \%$, severe degenerative AS with a mean gradient of $43 \mathrm{mmHg}$ and aortic valve area of $0.7 \mathrm{~cm} 2$ as well as moderate aortic regurgitation, moderate to severe mitral regurgitation and moderate tricuspid insufficiency with a systolic pulmonary artery pressure (sPAP) of $50 \mathrm{mmHg}$. His Society of Thoracic Surgery (STS) risk score ${ }^{[5]}$ for mortality was calculated as $14.8 \%$ for surgical aortic valve replacement (AVR). Heart valve team was consulted for evaluation for AVR and they decided to proceed with TAVR. A preoperative computed tomography angiography (CTA) was performed to decide the valve size and access site. Because the patient had ilio-femoral bypass surgery and had left SCA stent, the heart team decided to perform TAVR through the LAx. Although TAx TAVR can also be performed through the right axillary artery, the LAx was used in our patient as it provides a better angle for the advancement of the sheath toward the aortic root. The LAx was measured $6.5 \mathrm{~mm}$ at CTA. The patient was taken to the catheterization laboratory and the procedure was performed by a multidisciplinary team composed of interventional cardiologists, anesthesiologists and a cardiac surgeon. Valve replacement was carried out under general anesthesia. A 5F pigtail was first inserted percutaneously into the femoral artery and advanced to the level of the noncoronary sinus for aortic angiography. The third segment of the axillary artery (between the lateral border of the pectoralis minor and the inferior border of the teres major muscle) is the most distal segment of the axillary artery and can be preferred for percutaneous access due to a relative paucity of vascular branches. In our case, LAx was exposed at the third segment through a small incision at the medial aspect of the upper arm and secured with nylon tapes (Figure 1a). During the procedure, a bolus of intravenous heparin $(60 \mathrm{IU} / \mathrm{kg})$ was administered to achieve a target activated clotting time (ACT) of 250 to $300 \mathrm{~s}$ and the ACT was measured every 15 min thereafter. Then, the axillary artery was retracted with vessel loops, punctured and dilated. After direct insertion of a $6 \mathrm{~F}$ sheath (Figure 1b), a stiff wire was positioned in the ascending aorta and an $14 \mathrm{~F}$ sheath was then inserted through the LAx (Figure 1c). The rest of the procedure was carried out in a standard fashion. ${ }^{[6]}$ Briefly, the native valve was crossed retrogradely through the SCA stent (Figure 2a) cautiously (Video, Figure 2b) and dilated using balloon valvuloplasty (Figure 2c). The loaded prosthesis was then introduced through the 14F sheath and positioned across the aortic valve using angiographic and fluoroscopic guidance. When correct placement had been confirmed, a $26 \mathrm{~mm}$ S3 valve (Edwards Lifesciences, Irvine, CA) was deployed (Figure $2 \mathrm{~d}$ ). At this point, the patency of the coronary arteries and degree of aortic insufficiency were assessed using aortic angiography. The valve was also examined for the presence of any paravalvular leak. The wire and sheath were removed. The axillary artery is closed with paradoxal interrupted $5 / 0$ polypropylene sutures to avoid any stenosis and a wound suction device (HemoVac) is left in place to avoid compression of the surrounding tissues by an eventual hematoma. An angiogram of the LAx was performed at the end of each procedure and show the normal position and patency of SCA stent. The procedure was performed with 60 $\mathrm{ml}$ contrast media. Total procedure time was 54 minutes. Radiation dose and fluoroscopy time were 968 mGy and 26 minutes successively. After the procedure, the patient was taken to the coronary intensive care unit (ICU). Just 1 hour after the procedure, the patient developed ataxia and dysarthria. Head CT showed cerebellar ischemia due to an atheroembolism. Therefore, the patient was transferred to the neurology ICU for the aim of ischemic stroke treatment. Because the patient was already under dual antiplatelet therapy due to TAVR procedure, he was followed only under surveillance. After 3 days of surveillance, the patient was discharged from the hospital with no sequela.

\section{DISCUSSION}

Transcatheter aortic valve replacement is currently emerging as an alternative therapy for patients with severe AS at high risk for conventional AVR. ${ }^{[7]}$ Access route is one of the major determinant of success with TAVR. Presently, the access route of choice is transfemoral as vascular access can be achieved by percutaneous punction of the femoral artery or through a small surgical cutdown. However, the transfemoral approach is contraindicated in patients with small, tortuous or atheromatous ilio-femoral vessels, as it is associated with a high rate of procedural failure and vascular complications in these cases. ${ }^{\left[{ }^{8,9}\right]}$ In those patients with significant 
peripheral vascular disease, other alternatives include the TAx, transapical and transaortic approach.

Axillary artery is a good alternative access route for TAVR. Before choosing between right or left axillary artery, the clinician have to consider several factors. In general, the LAx is preferred as it allows for better co-axial orientation of the TAVR prosthesis in the aortic annulus. It also decreases the chance of carotid compromise relative to the right innominate artery. Other factors that should be considered include the aortic annular angle and the angulation of the takeoff of the subclavian/innominate artery from the aortic arch. An angle $>30^{\circ}$ between the annular plane and horizontal axis typically means a more difficult right-sided approach due to challenges achieving a co-axial approach. After evaluation of these factors, we decided to perform TAVR through LAx because of co-axial orientation, lots of TAVR experience through left subclavian artery, a number of ipsilateral transfemoral TAVR experience in patients with previous history of common/right or left iliac artery stenting and no experience of TAVR through right axillary artery. The exposure of the axillary artery is familiar to cardiac surgeons and is easily carried out through a small incision. Although the axillary and SCAs may be diseased, they are often good-sized and good-quality vessels even in patients who have atheromatous disease or extensive calcification affecting their ilio-femoral arterial systems. We were able to successfully implant the prosthesis in this patient. The patient had extensive atherosclerosis causing coronary and peripheral arterial disease. Therefore, he underwent successful TAVR with S3 bioprosthesis through the left axillary approach although there were left SCA stent, permanent pacemaker at left pectoral fossa and LIMA-LAD greft. Arı et al ${ }^{[10]}$ recently reported a TAx TAVR case in a patient with thoracic and abdominal multilayer flow modulator stents in addition to occluded bilateral carotid and iliac arteries.However, they used CoreValve prothesis instead of S3 valve used in our case.

In the literature S3 valve and the CoreValve Revalving System (Medtronic CV, Santa Rosa, CA) are available devices both of which have been successfully implanted through the axillary artery. Although, only the selfexpandable CoreValve has received the CE mark certification for this approach, we used balloon-expandable S3 valve due to the history of CABG, severe aortic calcification and high risk of paravalvular aortic regurgitaion. CoreValve bioprosthesis is not recommended in patients with CABG due to the risk of coronary ostia and bypass greft anastomosis blockage. Although this risk is low for S3 valve, we still measured the annulus to coronary ostia and proximal anastomosis distance before the procedure. The distance between aortic annulus and coronary ostia and proximal anastomosis was $11 \mathrm{~mm}$ and $15 \mathrm{~mm}$ successively. This distances was considered safe.

There is a few study and also a single center review related with TAx approach. Laflamme et al ${ }^{[11]}$ found that TAx approach for TAVR is safer and associated with less stroke, less vascular and bleeding complications compared to transfemoral and transapical route. However, in our case, the patient had mild atheroembolic stroke after the procedure. Moreover, the TAx approach has several potential advantages over the other implantation routes. Compared with the transfemoral technique, the shorter distance between the delivery sheath and the aortic annulus provides better control of the device and allows more precise positioning of the prosthesis. Although TAx TAVR can also be performed through the right axillary artery, the LAx was used in this patient as it provides a better angle for the advancement of the sheath toward the aortic root. This favorable angle, coupled with the superior control afforded by the position of the sheath close to the aortic valve, allowed easy positioning of the prosthesis in our case. The TAx approach also allows for less catheter manipulation than the transfemoral approach in the descending aorta and in the distal aortic arch, which may reduce the risk of embolic events secondary to displacement of atherosclerotic material. Furthermore, the fact that the prosthesis is advanced through the ascending aorta protected by the sheath may reduce the risk of atheromatous emboli. This theoretic advantage was not confirmed in our case as thromboembolic stroke occurred after the procedure. Surgical access of the axillary artery is frequently performed by cardiac surgeons as it is routinely cannulated for cardiopulmonary bypass during ascending aorta and aortic arch replacement. ${ }^{[12]}$ Therefore, access site complication is not frequently observed at TAx TAVR cases as in this case. The most important point for this successful TAx TAVR case was that the axillary artery caliber and aortic root was measured by the help of 3-D CTA and thus the suitability of axillary artery and SCA stent was determined during the passage of delivery system. Then, the procedure was performed carefully under flouroscopic guidance. 
In conclusion, TAVR using the LAx is a safe, reproducible and efficient procedure. It is an appealing alternative to the commonly used transfemoral approach in patients with severe iliofemoral disease and to the transapical approach in patients with severe associated lung disease. It can also be preferred in patients with ipsilateral SCA stent, permanent pacemaker and CABG with LIMA-LAD bypass greft after thoroughly reviewing the suitability of axillary artery for TAVR by the help of 3-D CTA. In addition, the interventional cardiologists should take care of SCA stent during passage of delivery catheter of TAVR to prevent the vessel rupture and stent stripping and should be alerted for the cessation of LIMA-LAD greft blood flow.

\section{CONFLICT OF INTERESTS}

The author declares that there is no conflict of interest.

\section{ETHICS STATEMENT}

Informed consent has been obtained from the patient for academic publication.

DATA AVAILABILITY STATEMENT: All data about this case is available

\section{REFERENCES}

1. Vemulapalli S, Carroll JD, Mack MJ, Li Z, Dai D, Kosinski AS, et al. Procedural volume and outcomes for transcatheter aortic valve replacement. N Engl J Med 2019;380: 2541-50.

2. Tomo A, Alexandros B, Sidakpal P. Advances in transcatheter aortic valve replacement. J Geriatr Cardiol 2019;16: 724-32.

3. Rajkumar D, Priyank S, Perwaiz M. In-hospital outcomes comparison of transfemoral vs transapical transcatheter aortic valve replacement in propensity-matched cohorts with severe aortic stenosis. Clin Cardiol 2018;41: 326-32.

4. Konstantinos VV, Chiu W, Ryan K, Polydoros NK, Andreas RB, Jonathan SW, et al. Transapical transcatheter aortic valve replacement in patients with or without prior coronary artery bypass graft operation. J Cardiothorac Surg 2016; 11: 158.

5. Webb J, Rodes-Cabau J, Fremes S, Pibarot P, Ruel M, Ibrahim R, et al. Transcatheter aortic valve implantation: a Canadian Cardiovascular Society position statement. Can J Cardiol 2012;28: 520-28.

6. Luigi B, Enrico F, Giovanni P, Francesco F, Tiziano M, Francesco P, et al. Access sites for TAVI: Patient selection criteria, technical aspects and outcomes. Front Cardiovasc Med 2018;5: 88.

7. Lindman BR, Bonow RO, Otto CM. Current management of calcific aortic stenosis. Circ Res 2013;113: $223-37$.

8. Murdock JE, Jensen H, Thourani V. Nontransfemoral approaches to transcatheter aortic valve replacement. Intervent Cardiol Clin 2015;4: 95-105.

9. Thourani VH, Li C, Devireddy C, Jensen HA, Kilgo P, Leshnower BG, et al. High-risk patients with inoperative aortic stenosis: use of transapical, transaortic, and transcarotid techniques. Ann Thorac Surg 2015;99: 817-23.

10. Arı H, Çamcı S, Karakuş A, Arı S, Melek M. Axillary artery as alternative access for TAVR in a patient with thoracic and abdominal multilayer flow modulator stents, and in a patient with occluded bilateral carotid and iliac arteries.Turk Kardiyol Dern Ars 2019;47: 399-405.

11. Laflamme M, Mazine A, Demers P, Lamarche Y, Ibrahim R, Asgar A, et al. Transcatheter aortic valve implantation by the left axillary approach: a single-center experience. Ann Thorac Surg 2014;97: 1549-54.

12. Laura S. Fong, Levi B, Manu N. Mathur. Liberal use of axillary artery cannulation for aortic and complex cardiac surgery. Interact Cardiovasc Thorac Surg 2013;16: 755-58.

\section{FIGURE LEGENDS}

Figure 1. A. Surgical cutdown of the third segment of left axillary artery at the medial aspect of the upper arm. B.placement of $6 \mathrm{~F}$ sheath. C. $14 \mathrm{~F}$ sheath insertion over a stiff guidewire (Amplatz Superstiff ST-1).

Figure 2. Fluoroscopy during percutanous transaxillary TAVR.A. Subclavian artery stent B. Passage of 
$14 \mathrm{~F}$ sheath through subclavian artery stent. C. Aortic balloon valvuloplastyy in addition to injection of a small amount of dye for sizing of the root anatomy. D. Edwards SAPIEN 3 valve immediately after its complete deployment.

Video: The cautiously crossed subclavian artery stent.
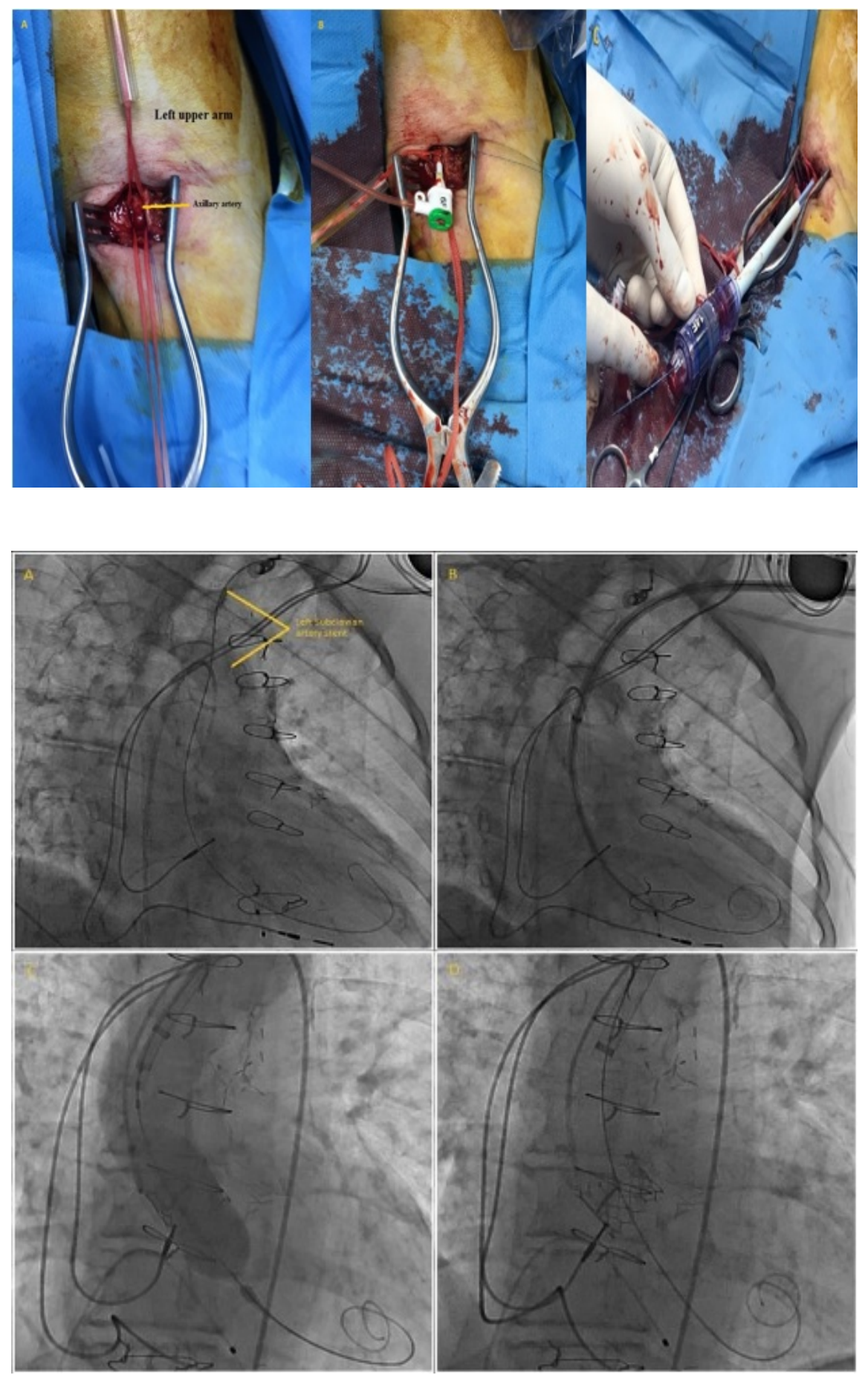